\title{
Psychological debriefing is a waste of time
}

\author{
SIMON WESSELY / MARTIN DEAHL
}

Edited and introduced by Mary Cannon, Kwame McKenzie and Andrew Sims.

\section{INTRODUCTION}

People who experience serious traumatic events may become distressed and are at risk of developing psychological illness. Because of the perceived need to ease the distress and to prevent chronicity, various forms of psychological therapy have been deployed. One such therapy is psychological debriefing. Some claim that it is helpful, others claim it may not do any good but at least it does no harm, but still others claim that it increases the risk of people developing long-term psychological symptoms following a traumatic event. Statutory agencies, charities and commercial organisations offer their services to victims following traumatic events. Fearing litigation, some companies require their employees to undergo debriefing following certain incidents. But is psychological debriefing the right treatment? Is it costeffective? And what of the concerns that it may lead to long-term problems? Could litigation be joined because of exposure to psychological debriefing? We asked two experts who have published widely on the subject - Professor Simon Wessely and Professor Martin Deahl - to debate the proposition that psychological debriefing is a waste of time.

\section{FOR}

Bad things happen to people. Sometimes these bad things cause long-standing psychological damage. The desire to reduce that impact is one of the laudable aspects of human nature. So it would be good news to report that not only do we in the mental health professions have the desire to prevent psychiatric disorder emerging in the aftermath of trauma, but we also have the ability to do so. Sadly we do not.

I take 'debriefing' to refer to some short, usually single-session, intervention that is performed with as many of those caught up in a traumatic event as possible, and involves some variation on the theme of going over the traumatic incident, linked with education about the expected emotional responses and assurances that these are normal. The rationale is to reduce acute emotional distress and prevent the onset of post-traumatic psychiatric disorder.

Debriefing is exceptionally popular - in a recent systematic review we identified over 50 different indications or uses, all of them involving trauma in some shape or form (Wessely et al, 2000). Many organisations offer debriefing as part of the organisational response to untoward incidents - such as police officers involved in firearms incidents, or bank staff who have been witness to robberies. In some such examples, interventions are compulsory perhaps out of a desire to reduce psychological distress, but also from a belief that this will reduce exposure to subsequent litigation.

There are many reasons why debriefing has flourished in recent years. When facing disasters, all of us must feel a need to do something. That talking about trauma must be better than 'repressing' or 'bottling-up' accords with a long and distinguished tradition in psychological treatment 'better out than in' - and has face validity.
Many people who have been debriefed report the experience in a positive fashion.

For some the virtues of debriefing are as obvious as the benefits of penicillin, and there has been resistance to submitting the process to what remains the only reliable method we have of knowing whether a treatment does more good than harm the randomised controlled trial (RCT). However, others, including many of those who were firm advocates of the procedure, have organised such studies and deserve considerable credit for so doing. The results of these studies have been summarised in several systematic reviews (e.g. Wessely et $a l, 2000)$.

These studies provide no evidence for any benefit of the intervention. All the modern studies fail to show any advantage to debriefing. But perhaps the most worrying findings come from the two trials scoring highest on the quality ratings, and with the longest follow-up times. The first reported the 18-month outcome of patients admitted to a Cardiff burns unit randomised to debriefing or no treatment (Bisson et al, 1997). There was a significant increase in the rates of post-traumatic stress disorder (PTSD) in those who had received the intervention. A persistent adverse effect of debriefing is also reported from the Oxford trial of debriefing of road traffic accident victims (Mayou et al, 2000).

Until these trials had been published, even to question the benefits of debriefing was difficult. However, armed with these results, we can now start to consider what might be going wrong. Perhaps the process of debriefing, part of the function of which is to warn participants of emotional reactions that might be expected to develop over weeks and months, actually increases the occurrence of these symptoms. Perhaps for some not talking is indeed appropriate defence mechanisms may serve a purpose, and it is not always 'better out than in'. Talking to a stranger, whom one has never met before and will not meet again, may impede the normal processes of recovery that utilise one's own social networks family, friends, general practitioner and others who may be better able to place the trauma in the context of one's own life. Perhaps debriefing acts to professionalise distress, part of the general process of the professionalisation of adversity across society.

Not everyone will accept these findings. It is inevitable that when a cherished belief is challenged, various counterclaims are 
made - the evidence is for the wrong type of debriefing, the trials were not well done, elements of debriefing could still 'work', the testimonies of those who are certain it helped them cannot be discounted etc. but this should not distract us from the main findings. We should also beware of the tendency to say, 'OK, let's forget about debriefing, but defusing - that's something different and that does work'. I am afraid I cannot exactly see the differences between the various forms of debriefing, and I can find no serious argument as to why one should be ineffective, whereas something that seems to be a variation should be effective, even though there is no evidence in support of the proposition.

What is now clear is that it is time to urgently reassess how we respond to trauma. There can be no doubt that those who are attempting to help people involved in disasters and trauma have noble motives, but that sadly is not enough. Instead, it is time that at the very least those who are asked to take part in debriefing, which by definition will happen when people are vulnerable, are warned that the process has the capacity to do harm as well as good. Compulsory debriefing, which is still the case in some organisations, must cease forthwith. Perhaps it is also time to consider whether attempts to prevent the onset of PTSD are premature, and resources might be better spent on treating those who later develop psychiatric disorder, for whom there are now recognised treatments but grossly inadequate resources to meet the need.

I am not arguing that all forms of early support and intervention should cease. Instead, I draw attention to two promising developments. First, the studies of Richard Bryant in Australia on trauma survivors where the intervention was not offered to everyone, but only to the minority with acute stress disorders, who are at higher risk of developing subsequent psychiatric disorder. The intervention was not singlesession but multiple-session and was based on a coherent cognitive-behavioural model (Bryant et al, 1999). The second is the work of Captain Cameron March. Surmising that a key problem with debriefing is the use of strangers unknown to the participants, and invariably coming from another culture or organisation, Captain March has initiated brief and simple training for all ranks of the Marines. The rationale is that when a traumatic event happens to a unit, then those members of the unit will be able to offer each other a common-sense, lowkey, supportive intervention that is firmly rooted in organisational culture, and involves no outsiders and no risk of 'professionalising' normal distress.

The story of debriefing teaches salutary lessons. First, people are more resilient than we given them credit for. Second, although it is indisputable that major progress has been made in the management of PTSD, prevention, as in the rest of psychiatry, is an area fraught with difficulty. No matter how well-meaning are our attempts to reduce distress and prevent psychiatric disorder, and no matter how self-evident is the intervention, we still require firm evidence of benefit, and must remember that any health care intervention always has the capacity to do harm as well as good, and occasionally the balance between the two will continue to surprise us.

S. Wessely Institute of Psychiatry, De Crespigny Park, London SE5 8AF, UK. E-mail:

s.wessely@iop.kcl.ac.uk

\section{AGAINST}

Psychological debriefing was designed for groups of individuals exposed to traumatic events to improve their emotional wellbeing at the time and to prevent or minimise subsequent mental disorder (particularly PTSD). The effectiveness of psychological debriefing and other early interventions remains one of the most contentious areas of mental health research.

The debate raises issues of fundamental importance for psychiatry. It has also become highly politicised as disasters and high-profile compensation claims for psychological injury have cast the 'debriefing debate' into the public arena. Considering that the prevalence of PTSD (notwithstanding a variety of other post-traumatic disorders) is said to be $2-3 \%$, the effectiveness of early intervention is also a major public health issue.

The past 15 years have spawned a 'disaster industry' and diverse groups, including statutory organisations and the commercial and voluntary sector, have at times uncritically promoted psychological debriefing following traumatic events. Dialectically opposed is the 'anti-therapy' movement that has drawn unjustified parallels between psychological debriefing, counselling and psychotherapy, generalising the results of trials of psychological debriefing in order to challenge the evidence base of those therapies. However, it is unfair and deceptive to generalise the findings of debriefing research (a preventive measure for healthy individuals) to the psychological treatment of established disorder and disparate forms of counselling in diverse settings such as marital breakdown, bereavement, rape and child sexual abuse.

Although intuitively appealing and a response to perceived need, demonstrating the effectiveness of any early intervention has proved difficult and it is only recently that psychological debriefing has been subject to randomised controlled clinical trials. The 2002 Cochrane review included 11 studies comparing psychological debriefing with 'no-intervention' controls and concluded that psychological debriefing was of no value in preventing PTSD. Indeed, two trials found that it actually made subjects symptomatically worse (Rose et al, 2002). The studies on which these conclusions were based, however, have little to do with psychological debriefing in the real world and comprised single-session 'one-off' debriefing of single subjects (not selected from epidemiological samples) who were medically ill (or obstetric patients). Symptomatology of PTSD was generally employed as the sole outcome measure, although it is recognised that PTSD is but one of a number of post-traumatic syndromes. None of the studies assessed the impact of psychological debriefing on other important consequences of trauma such as alcohol and substance misuse, or its effect on social or occupational functioning.

The observation that psychological debriefing worsens symptoms is frequently cited by its opponents. They fail to mention, however, that the two RCTs that suggest that psychological debriefing may be harmful both failed to achieve equivalent group membership at pre-test (debriefed groups had more severe injuries in both studies) (Hobbs et al, 1996; Bisson et al, 1997). These pre-test differences may well have influenced post-intervention outcomes. Moreover, the deterioration in psychopathology of the debriefed group in one of these studies, although statistically significant, was so slight as to be clinically irrelevant (Hobbs et al, 1996).

Interestingly, the Cochrane review explicitly excluded a further 19 studies because of 'methodological shortcomings', principally concerning problems of randomisation. These included RCTs of group debriefing in the naturalistic settings for which psychological debriefing was 
intended. Some of these measured a broader range of outcome measures and, interestingly, some demonstrated a positive debriefing effect on these (e.g. Deahl et al, 2000).

The conclusions of the Cochrane review and its exclusions raise important questions about the nature of evidence and the status of RCTs as the imprimatur of good evidence. Level I evidence - RCTs - have become the dominant paradigm of treatment outcome studies to the virtual exclusion of naturalistic, observational studies or case series (evidence levels II-V). Conducting a methodologically rigorous RCT of group debriefing is, however, particularly challenging given that group trauma (for which psychological debriefing was intended) occurs only in unpredictable and often chaotic circumstances such as armed conflict or disaster where the operational imperative is paramount. Despite methodological shortcomings, particularly relating to sample selection and randomisation to different treatment conditions in conflict, following disaster or post-accident, naturalistic studies, often conducted opportunistically, remain useful and have considerable heuristic value. In attempting to satisfy the rigorous methodological criteria demanded of level I evidence, many RCTs loose validity and become so divorced from clinical reality that their findings are clinically meaningless. Level I RCTs are not the sine qua non of evidence-based medicine; psychological debriefing research, which challenges their hegemony and lends credibility to observational studies, has important implications for the ways in which we judge the quality and value of research.

Whether or not psychological debriefing reduces long-term morbidity, several studies report that individuals find it subjectively helpful at the time (although this is another outcome that has not been properly studied). Under these circumstances can it, therefore, be ethically justifiable to employ non-intervention controls, denying individuals short-term support, whatever the long-term outcome? For an operational commander on the battlefield or at a disaster site, 'feeling better' at the time may be a desirable outcome irrespective of any longer-term benefits.

Although it seems clear at this point that there is insufficient evidence to recommend offering one-off single-session debriefing or crisis counselling to medical patients, this finding has very little to do with the task of addressing the mental health needs of victims in the wake of a mass disaster. Whatever its intrinsic benefit, psychological debriefing provides an opportunity to focus on the psychological welfare of trauma victims. It has an important educational role and allows an opportunity to identify individuals suffering from acute stress reactions (who are at greater risk of developing longer-term disorders). Singlesession psychological debriefing may well do harm, not by any direct effect on mental state but rather by fostering an air of complacency (in assuming that an individual who has had debriefing will be immune from subsequent disorder). It may also damage and make secondary victims of the 'debriefers' who themselves require adequate support and supervision. Psychological debriefing was never intended to be a stand-alone intervention - rather it should be but one part of a comprehensive stress management package that enables individuals to receive follow-up, an assessment of individual need and practical support, as well as allowing the early detection and prompt treatment of established
PTSD and other disorders. Abandoning psychological debriefing sends out the dangerous message that doing nothing for individuals following traumatic events is acceptable.

M. Deahl Shropshire County Primary Care Team (Shelton Hospital, Bicton Heath, Shrewsbury SY3 8DN, UK) and Visiting Professor, City University, London. E-mail: martindeahl@fsmail.net

\section{REFERENCES}

Bisson, J. I., Jenkins, P. L., Alexander, J., et al (1997) Randomised controlled trial of psychological debriefing for victims of acute burn trauma. British journal of Psychiatry, I7I, 78-8I.

Bryant, R., Sackville, T., Dang, S., et al (1999)

Treating acute stress disorder: an evaluation of cognitive behavior therapy and supportive counselling technques. American Journal of Psychiatry, 156, 1780-1786.

Deahl, M., Srinivasan, M., Jones, N., et al (2000)

Preventing psychological trauma in soldiers. The role of operational stress training and psychological debriefing. British Journal of Medical Psychology, 73, 77-85.

Hobbs, M., Mayou, R., Harrison, B., et al (1996) A randomised controlled trial of psychological debriefing for victims of road traffic accidents. BM/, 3/3, 1438-1439.

Mayou, R., Ehlers, A. \& Hobbs, M. (2000)

Psychological debriefing for road traffic accident victims: three year follow-up of a randomised controlled trial. British Journal of Psychiatry, I76, 589-593.

Rose, S., Bisson J. \& Wessely S. (2002) Psychological debriefing for preventing post-traumatic stress disorder Cochrane Library, issue 2. Oxford: Update Software.

Wessely, S., Bisson, J. \& Rose, S. (2000) A systematic review of brief psychological interventions ('debriefing') for the treatment of immediate trauma related symptoms and the prevention of post traumatic stress disorder. In Depression, Anxiety and Neurosis Module of the Cochrane Database of Systematic Reviews (eds M. Oakley-Browne, R. Churchill, D. Gill, et al). Oxford: Update Software. 